\title{
Morphologische Parameter als Biomarker in der Ökotoxikologie - natürliche und schadstoffinduzierte Variabilität
}

\author{
Helmut Segner
}

Eingegangen: 19. Dezember 2008 / Akzeptiert: 24. Februar 2009/Online veröffentlicht: 20. März 2009

(C) Springer-Verlag 2009

Zusammenfassung Hintergrund Veränderungen in der Ausprägung biologischer Merkmale von Organismen werden in der Ökotoxikologie als Biomarker genutzt, um die Exposition von Organismen an toxische Stoffe respektive die Wirkung toxischer Stoffe nachzuweisen. In der Umweltbeobachtung können Biomarker als sensible Indikatoren für die chemische Belastung eines Lebensraumes und als frühzeitiger Hinweis auf mögliche adverse Effekte dienen.

Ziel Voraussetzung zur Nutzung biologischer Merkmale als Biomarker ist die Fähigkeit, die natürliche oder ,normale“ Variabilität der Merkmalsausprägung eindeutig von induzierten oder abnormalen Veränderungen abgrenzen zu können. Der Beitrag hat zum Ziel, mögliche Probleme bei der Abgrenzung von ,normal“" und ,verändert“ am Beispiel morphologischer Biomarker deutlich zu machen.

Ergebnisse und Diskussion Morphologisch-anatomische Merkmale von Organismen werden oft intuitiv als invariabel empfunden. Am Beispiel der Gonadenmorphologie von Fischen, die als Biomarker für die Wirkung hormonaktiver Stoffe eingesetzt wird, zeigt der Beitrag, dass auch morphologische Parameter eine ausgeprägte Grundvariabilität aufweisen können. Die Ursachen dieser Variabilität sind häufig nicht verstanden. Dies schränkt die Interpretierbarkeit von Biomarkerbefunden stark ein, insbesondere wenn die Organismen neben der Belastung mit toxischen Chemikalien weiteren Stressoren ausgesetzt sind.

Ausblick Das Problem der Kenntnis und Interpretierbarkeit der Grundvariabilität von Biomarkern ist besonders in je-

Herrn Prof. Dr. Dr. h.c. Volker Storch zum 65. Geburtstag gewidmet. Herausgeber: Henner Hollert • Thomas Braunbeck

H. Segner $(\bowtie)$

Zentrum für Fisch- und Wildtiermedizin, Vetsuisse Fakultät,

Universität Bern, Postfach 8466, 3001 Bern, Schweiz

E-Mail: helmut.segner@itpa.unibe.ch nen Situationen relevant, in denen die chemische Belastung nicht der dominierende Stressfaktor ist, sodass die chemikalieninduzierte Veränderung der Biomarker leicht von anderen Einflüssen überlagert werden kann. Zur Interpretation derartiger Situationen sollte die Ökotoxikologie vermehrt die phänotypische Plastizität von Organismen und Genotypen mit einbeziehen.

Schlüsselwörter Biomarker · Endokrine Disruption · Fisch · Gonadenveränderungen · Morphologie · Phänotypische Plastizität · Variabilität

\section{Morphological parameters to be used as biomarkers in ecotoxicology - natural and induced variability}

Abstract Background Ecotoxicology utilizes alterations of biological parameters of organisms as biomarkers of toxic exposure or effects. In environmental monitoring, biomarkers function as sensitive indicators of chemical pollution or as early warning signal of late effects

Aim The pre-requisite for using a biological parameter as biomarker is the ability to unequivocally distinguish between the natural or normal and the induced or abnormal expression of the marker. This article discusses problems in discriminating between the normal and induce state, using morphological biomarkers as an example.

Results and Discussion Morphological and/or anatomical parameters are intuitively considered to be rather invariable. This article shows for the example of gonad morphology of fish that this expectation is not always correct, but that morphological markers may display pronounced baseline variability. The reasons for this variability are often not understood. This is limiting the utility and interpretation of the biomarker response, in particular when 
organisms are not only exposed to chemical but to multiple stressors.

Outlook The problem of discriminating between natural and induced variability of biomarkers is of particular relevance in low dose exposure scenarios, when stressors other than toxic chemicals may confound the toxicant-induced changes of the biomarker status. To better handle this problem and to be able to distinguish between adaptive and adverse changes, ecotoxicology has to set out for improved understanding of the phenotypic plasticity of organisms and genotypes,

Keywords Biomarker - Endocrine disruption - Fish · Gonad pathology $\cdot$ Morphology $\cdot$ Phenotypic plasticity Variability

\section{Problemstellung}

Die Ökotoxikologie nutzt Veränderungen in der Ausprägung biologischer Merkmale als Indikatoren oder Marker, um die Exposition von Organismen gegenüber chemischen Stoffen zu charakterisieren bzw. die Wirkung der chemischen Exposition zu diagnostizieren. Ein Beispiel ist die Induktion von Vitellogenin als Biomarker für eine Exposition an Östrogen-wirksame Stoffe. Implizite Voraussetzung für diesen Ansatz ist es, dass eindeutig zwischen der ,normalen“ oder „natürlichen“ Ausprägung der Merkmale - in Abwesenheit des Stressors - und dem induzierten Zustand unterschieden werden kann. Mit den Worten von Sumpter und Johnson (2005): „It is axiomatic that it is not possible to conclude something is abnormal unless one knows what is normal". Um biologische Merkmale für die Erfassung, Beobachtung und Bewertung chemischer Umweltbelastungen zu nutzen, ist es also erforderlich, deren Variationsbreite im ungestörten, nicht durch einen Stressor beeinflussten $\mathrm{Zu}$ stand (Homöostase) zu kennen; nur dann kann eindeutig und zuverlässig die natürliche oder „,normale“ Variabilität von der induzierten oder abnormalen Veränderung abgegrenzt werden. Obwohl diese Anforderung auf den ersten Blick trivial erscheinen mag, kann sie in der Umsetzung durchaus schwierig sein. Ein Problem ergibt sich aus der Tatsache, dass wir es in der Ökotoxikologie mit sehr vielen Arten zu tun haben, und die Kenntnisse zur Hintergrundvariabilität der biologischen Merkmale bei vielen Arten sehr begrenzt sind. Selbst für die in den ökotoxikologischen Labortests genutzten Modellarten ist das Wissen um die natürliche Variabilität von biologischen Eigenschaften oft sehr lückenhaft (Watanabe et al. 2007; Segner 2009). Ein zusätzliches Problem ergibt sich aus der Tatsache, dass nach Exposition von Organismen gegenüber toxischen Stoffen Organismen in der Regel nicht in Form einer Ja/Nein-Antwort, sondern graduell vom Normalzustand in den veränderten Zustand übergehen (Walker 1998). Dies führt zu der Frage, wel- ches Ausmass an Modifikation einer Merkmalsausprägung noch im Bereich der natürlichen Schwankungen liegt, d.h., $a b$ wann eine über den Normalbereich hinausgehende, umweltinduzierte Veränderung der Merkmalsausprägung vorliegt. Nach dem klassischen Dosis-Wirkungs-Modell in der Toxikologie fällt die Auslenkung aus dem Normalbereich umso stärker und eindeutiger aus, je höher die Konzentration des Stoffes ist. Im Laborversuch kann im Falle quantitativ erfassbarer Indikatorparameter die Trennung „,normal“ versus ,verändert“ in der Regel innerhalb einer Konzentrations-Wirkungs-Kurve anhand des statistischen Vergleichs der exponierten Gruppe mit einer Kontrollgruppe durchgeführt werden - wobei Ausnahmen existieren, z.B. DosisWirkungs-Beziehungen in Form einer Optimumskurve. In Freilanduntersuchungen ist eine Trennung von normaler Variabilität und induzierten Veränderungen oft schwieriger: einerseits, weil klar definierte Kontrollgruppen oft fehlen und andererseits, weil die chemikalieninduzierte Reaktion der Indikatorparameters durch die Einwirkung anderer Stressoren überlagert und modifiziert sein kann. Als Beispiel hierzu sei die durch Temperaturschwankungen bedingte Veränderung des Biomarkers Cytochrom P4501A genannt. Die Expression dieses Xenobiotika-metabolisierenden Enzyms wird durch dioxinähnliche Chemikalien einschliesslich polychlorierter Biphenyle und polyaromatischer Kohlenwasserstoffe induziert und ist in der Ökotoxikologie einer der am meisten genutzten Expositionsbiomarker. Bei Fischen wird die Expression von Cytochrom P4501A jedoch nicht nur durch Chemikalienexposition, sondern $u$. a. auch durch die Wassertemperatur beeinflusst. Tatsächlich haben Freilanduntersuchungen gezeigt, dass Veränderungen der Wassertemperatur die chemikalienbedingte Induktion von $\mathrm{Cy}$ tochrom P4501A überlagern und maskieren können (Lange et al. 1998; Behrens und Segner 2005). Um Biomarker angesichts solcher multipler Regulationen dennoch als Indikatoren für Chemikalieneffekte einsetzen zu können, werden in Freilanduntersuchungen häufig Ansätze gewählt, bei denen die Ausprägung der Biomarkerantworten in räumlichen (z.B. Längsprofile, Transekte) oder zeitlichen Gradienten („Before-After-Control-Impact“, BACI) mit Unterschieden in der chemischen Belastung verglichen werden, um aus diesem Vergleich korrelativ auf einen Zusammenhang zwischen der Merkmalsausprägung und der Belastung zu schliessen (Underwood 1994; Downes et al. 2002).

Ziel des vorliegenden Beitrags ist es, die Bedeutung der Frage ,was ist normal“ resp. „was ist verändert/induziert“ im Hinblick auf die ökotoxikologische Risiko- und Umweltbewertung zu diskutieren. Die Betrachtung wird sich auf das Beispiel anatomisch-morphologischer Merkmale beschränken. Veränderungen in makroskopischen und mikroskopischen Strukturmerkmalen werden häufig als Expositions- oder Wirkungsbiomarker genutzt (Hinton et al. 1987; Schwaiger et al. 1997; Braunbeck 1998; Au 2004). Ein bekanntes Beispiel 
für den Einsatz morphologischer Endpunkte als Expositionsmarker ist die durch Tributylzinn induzierte Bildung von Imposex bei Mollusken (Oehlmann et al. 1996). Im Sinne von Effektmarkern sind die durch verschiedene Umweltschadstoffe bedingten Neoplasien bei Fischen einzuordnen (Myers et al. 1994; Köhler 2004). Insbesondere bei freilebenden Tierpopulationen ist jedoch die Grundvariabilität anatomischmorphologischer Merkmale oft nur unzureichend bekannt oder wird unterschätzt. Im Folgenden soll diese Problematik anhand weniger Beispiele diskutiert werden.

\section{Wie konstant oder variabel sind anatomisch-morphologische Merkmale?}

Im Gegensatz zu molekularen und biochemischen Parametern werden anatomische und morphologische Strukturen intuitiv oft als konstant oder wenig variabel wahrgenommen. Diese Sichtweise findet ihren Niederschlag beispielsweise in der evolutionsbiologischen Forschung, bei der aus dem Vergleich der Morphologie von Zellen und Organen phylogenetische Beziehungen abgeleitet werden (Eakin 1972; Storch 1979; Jamieson 1986; Storch und Welsch 1989). Trotz dieser Konstanz distinkter Strukturen darf man jedoch nicht übersehen, dass bestimmte Zellen und Organe ein hohes Mass an morphologischer Variabilität unter dem Einfluss endogener oder exogener Faktoren aufweisen können. In welch überraschend starkem Masse Umweltfaktoren die Morphologie von Zellen und Organen beeinflussen können, haben die Arbeiten von Volker Storch deutlich gemacht (Storch 1982; Storch et al. 1983). Die Experimente der Arbeitsgruppe Storch zur Wirkung von Nahrungs- und Schadstoffstress auf die zelluläre Variabilität bei Wirbellosen und Wirbeltieren haben in eindrücklicher Weise gezeigt, wie eng Zellausgestaltung und Umweltfaktoren korrelieren, und dass aufgrund dieser Abhängigkeit die Morphologie von Zellen als „Spiegel der Umwelt“ dienen kann (Storch 1985).

Morphologische Variabilität ist Teil der phänotypischen Plastizität, mit der Organismen auf Umweltfaktoren reagieren. Beispiele für umweltinduzierte morphologische Plastizität sind z. B. durch Prädatoren ausgelöste Veränderungen von Körperformen bei Beutespezies (McIntyre et al. 2004), durch physisches Training ausgelöste Veränderungen der Muskelstruktur (Johnston 2006), oder durch Temperaturund Nahrungsschwankungen induzierte morphologische Veränderungen in zentralen Stoffwechselorganen wie der Leber bei Wirbeltieren oder der Mitteldarmdrüse bei Wirbellosen (Braunbeck et al. 1987; Segner und Möller 1984; Vogt et al. 1986; Dittbrenner et al. 2008). Entsprechend führt auch die Belastung mit toxischen Stoffen zu morphologischen Veränderungen in Zielorganen und -zellen (Au 2004; Braunbeck et al. 1989; Braunbeck 1998; Burkhardt-Holm et al. 1998; Gernhöfer et al. 2001; Hinton et al. 1978, 2008; Köhler et al. 1992; Triebskorn und Künast 1990; Vogt 1987). Die Induktion morphologischer Veränderungen durch exogene Faktoren kann einerseits über eine Beeinflussung der Morphogenese erfolgen, wie es beispielsweise für die Wirkung von hormonaktiven Stoffen auf die Differenzierung der Gonadenmorphologie (s.u.), oder für die durch Umweltstressoren bedingte fluktuierende Asymmetrie anatomischer und morphologischer Merkmale gezeigt wurde (Clarke 1993; Brakefield 1997; Söderman et al. 2007). Andererseits sind exogene Faktoren in der Lage, die Morphologie bereits ausdifferenzierter Zellen und Organe zu verändern. Beispielswiese führt die Exposition von juvenilen oder adulten Fischen gegenüber toxischen Wasserinhaltsstoffen zu strukturellen Veränderungen sowohl in den Kiemen - als dem Organ, mit dem wassergelöste Substanzen zuerst in Kontakt kommen - wie in jenen inneren Organen, die in die Akkumulation, die Verteilung, die Metabolisierung und die Exkretion der Stoffe involviert sind (Couch 1975; Hinton et al. 1987; Segner 1987; Braunbeck et al. 1989). Umweltinduzierte morphologische Reaktionen können schnell erfolgen, innerhalb von Stunden oder wenigen Tagen (Böhm et al. 1994).

In der Ökotoxikologie wird die primär qualitative Natur morphologischer Parameter oft als Nachteil für deren Nutzung als Biomarker empfunden. Jedoch sind auch strukturelle Merkmale grundsätzlich einer Quantifizierung zugänglich, sei es über semiquantitative Auswertungsschemata (Bernet et al. 1999) oder über quantitative stereologische Methoden (Segner und Braunbeck 1990). Allerdings gilt auch in diesem Fall die oben formulierte Forderung, dass, um eine umweltinduzierte Veränderung diagnostizieren zu können, die natürliche Variabilität des Markers hinreichend gut bekannt sein muss.

\section{Veränderungen der Gonadenmorphologie und -anatomie von Fischen als Biomarker für Exposition gegenüber hormonaktiven Stoffen: Intersex-Gonaden}

In der aquatischen Umwelt treten eine Reihe von Stoffen auf, die modulierend oder advers in das Hormonsystem von exponierten Organismen eingreifen können (Sumpter und Johnson 2005). Diese sogenannten hormonaktiven Stoffe oder endokrinen Disruptoren umfassen eine diverse Gruppe von chemischen Substanzen, die von Industriechemikalien wie z. B. Bisphenol A über Pharmazeutika wie z. B. Äthinylöstradiol bis zu den von Mensch und Tier ausgeschiedenen natürlichen Hormonen reichen. Wichtige Eintragswege der hormonaktiven Stoffe in die Gewässer sind Kläranlagen. Hormonaktive Stoffe entfalten ihre Wirkung entweder durch eine Störung des Hormonstoffwechsels, also Synthese, Transport, Abbau und Exkretion, oder durch agonistische resp. 
antagonistische Bindung an endogene Hormonrezeptoren. Besondere Aufmerksamkeit unter den endokrinen Disruptoren haben östrogenaktive Stoffe gefunden, das sind Stoffe, die an die Östrogenrezeptoren von Wirbeltieren binden, und über eine Aktivierung der Östrogenrezeptoren Störungen im Sexualsteroidsystem und letztlich in der Sexualentwicklung und der Reproduktion auslösen (McLachlan 2001).

Als Pionierstudien, die auf die Kontamination der aquatischen Umwelt mit hormonaktiven Substanzen aufmerksam machten, gelten die Untersuchungen zum Auftreten von „Intersex“-Gonaden bei Rotaugen, Rutilus rutilus, in englischen Flüssen (Jobling et al. 1998). Intersex-Gonaden zeichnen sich durch das gleichzeitige Auftreten von männlichen und weiblichen Keimzellen in den Gonaden von normalerweise getrenntgeschlechtlichen Tierarten aus (Dittrich et al. 2009). Dabei finden sich entweder weibliche Keimzellen in einer überwiegend männlich differenzierten Gonade (Abb. 1) oder männliche Keimzellen in einer überwiegend weiblich differenzierten Gonade (Vine et al. 2005; Körner et al. 2007). Jobling et al. (1998) beobachteten in ihrer Studie, dass in bestimmten Flüssen bis zu $100 \%$ der Rotaugen bei männlich differenzierten Gonaden zusätzlich Eizellen enthielten, also Intersex-Gonaden aufwiesen. Die Häufigkeit der Oozyten im Hodengewebe variierte individuell, vom Auftreten einzelner Oozyten bis zu einem sehr hohen

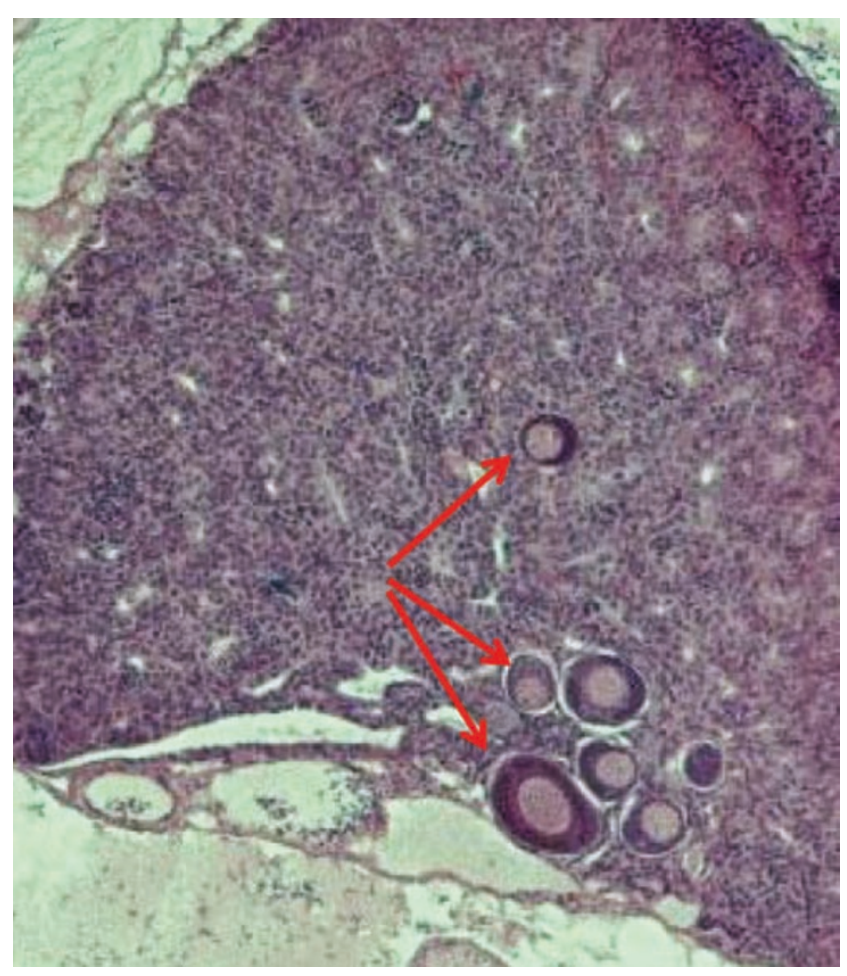

Abb. 1 Intersex-Gonaden von Bachforellen. Im Gonadengewebe mit testikulärer Morphologie finden sich vereinzelte, unreife Oozyten (Pfeile)
Anteil. Die Prävalenz von Fischen mit Intersex-Gonaden in den untersuchten Populationen korrelierte mit der Einleitung von Kläranlagenabwässern: Unterhalb von Kläranlagenausläufen fanden sich signifikant mehr Rotaugen mit IntersexGonaden als oberhalb der Ausläufe. Die Ursache für das gehäufte Auftreten von Intersex-Fischen in den englischen Flüssen sah man in der Präsenz östrogenaktiver Substanzen in den Kläranlagenausläufen (Desbrow et al. 1998). Diese Interpretation wurde von Laborstudien unterstützt, die nachwiesen, dass die Exposition von Fischen gegenüber östrogenaktiven Substanzen zu einer konzentrationsabhängigen Induktion der Intersex-Morphologie führen kann (Gray und Metcalfe 1997; Gimeno et al. 1998; Kipfer et al. 2009).

Bei einer hohen Prävalenz und einer starken Ausprägung des morphologischen Merkmals „Intersex“ in einer freilebenden Fischpopulation, so wie es in der Studie von Jobling et al. (1998) der Fall war, ist die Annahme, dass hier ein abnormales, durch Exposition an einen Umweltstressor induziertes Phänomen vorliegt, vernünftig. Schwierig wird die Interpretation jedoch beim Vorliegen eines relativ niedrigen Anteils und einer schwachen Ausprägung von Intersex; in einer solchen Situation ist es schwierig zu entscheiden, ob eine natürlicherweise vorhandene Hintergrundvariabilität in der Gonadenmorphologie der betrachteten Art vorliegt oder ob es sich um eine durch Exposition gegenüber endokrinen Disruptoren induzierte morphologische Reaktion handelt (van Aerle et al. 2001; Gerken and Sordyl 2002; Sumpter und Johnson 2005). In der Literatur gibt es eine Reihe von Berichten, die das gelegentliche Auftreten von Intersexualität bei getrenntgeschlechtlichen (gonochoristischen) Fischarten beschreiben (Brown und Scott 1988; Kinnison et al. 2000). Vine et al. (2005) fanden bei $14 \%$ von 112 Hechten (Esox lucius) aus englischen Gewässern Intersex-Gonaden, wobei die Prävalenz unabhängig davon war, ob die Hechte unterhalb oder oberhalb von Kläranlagen gefangen wurden. Körner et al. (2007) berichteten, dass 5\% der von ihnen untersuchten 424 Bachforellen (Salmo trutta) aus Schweizer Gewässern Intersex-Gonaden aufweisen. In beiden Studien konnten die Autoren auf der Basis des vorhandenen Wissens zur Intersex-Häufigkeit bei freilebenden Fischarten nicht entscheiden, ob die beobachteten niedrigen Prävalenzen einem natürlichen Hintergrundwert entsprechen, oder ob sie ein durch hormonaktive Stoffe induziertes Phänomen darstellen. Gradientenansätze, wie in der Studie von Vine et al. (2005), können in der Interpretation der Beobachtungen weiterhelfen, sind jedoch nicht immer zielführend, insbesondere wenn, wie häufig in Freilandstudien der Fall, die Stichprobengrösse gering ist (Sumpter und Johnson 2005).

Die obigen Beispiele zeigen, wie wichtig es ist, mehr Grundlagenwissen zur natürlichen Merkmalsvariabilität bei freilebenden Wildtierpopulationen zu generieren, um biologische Merkmale von Arten als Marker für die Exposition gegenüber resp. die Wirkung von Umweltstressoren 
einsetzen zu können. Aber selbst für die in Laboruntersuchungen vielfach verwendeten „Modellarten“ ist das Wissen zur natürlichen Variabilität oft recht limitiert (Watanabe et al. 2007). Dies wird deutlich anhand einer retrospektiven Studie (Grim et al. 2007) zur Häufigkeit von Intersex beim Medaka (Oryzias latipes), einer der drei Fischarten, die von der OECD zur Durchführung von Labortests auf endokrin wirksame Stoffe vorgeschlagen sind. Die Auswertung von 41 toxikologischen Studien aus drei unterschiedlichen Laboratorien ergab, dass in 36\% der Studien Fische aus den Kontrollgruppen Intersex-Gonaden aufwiesen. Die Prävalenz von Intersex-Individuen in den Kontrollgruppen der 41 Studien variierte von 0 bis $100 \%$.

\section{Variabilität der Gonadenmorphologie bei Felchen aus dem Thuner See: normal oder induziert?}

Als ein weiteres Beispiel für die Problematik der Abgrenzung natürlicher und induzierter Variabilität morphologischer Merkmale bei freilebenden Wildtierpopulationen sollen die Gonadenveränderungen von Felchen (Coregonus lavaretus) aus dem Thuner See, einem voralpinen See in der Schweiz, diskutiert werden. Erstmals im Jahre 2000 wurden von kommerziellen Fischern am Thuner See eigenartige Veränderungen der Gonadenmorphologie von Felchen beobachtet. Eine daraufhin eingeleitete detaillierte Untersuchung zeigte, dass sich verschiedene Kategorien an Veränderungen unterscheiden liessen, u. a. eine Kompartimentierung der Gonaden in einzelne Fragmente, Asymmetrien zwischen linker und rechter Gonadenhälfte, Verwachsungen der Geschlechtsorgane mit der lateralen Muskulatur oder die Ausbildung von Zwittergo- naden (Bernet et al. 2004). Die Prävalenz der Gonadenveränderungen lag bei $35 \%$. In der Literatur existieren zwar einige Berichte zum Auftreten von morphologischen Gonadenvariationen bei Felchen (Literatur siehe Bernet et al. 2004), in all diesen Berichten handelte es sich jedoch um einzelne oder wenige Fische innerhalb einer Population; in keinem Fall wurden vergleichbare Prävalenzen berichtet wie für die Felchen des Thuner Sees. Auch eine intensive Umfrage unter Wissenschaftlern brachte keinerlei Hinweise auf eine natürlicherweise vorhandene hohe Variabilität der Gonadenmorphologie von Felchen. Diese Datenlage legte die Annahme nahe, dass es sich im Falle der Thuner-See-Felchen nicht um eine natürliche Hintergrundvariabilität, sondern um ein induziertes Phänomen handelt. Als möglicher Ursachenfaktor standen endokrine Disruptoren im Vordergrund (Bernet et al. 2008), da für diese Wirkgruppe in einer Reihe von Studien ein Zusammenhang zwischen Exposition und dem Auftreten morphologisch veränderter Gonaden bei Fischen gezeigt wurde.

Eine epidemiologische Analyse der Art und Häufigkeit der Gonadenveränderungen bei Felchen aus dem Thuner See im Vergleich zu Felchen aus zwei weiteren voralpinen Seen - Bieler See und Brienzer See - zeigte jedoch, dass die Grundvariabilität der Gonadenmorphologie von Felchen bisher klar unterschätzt wurde (Bittner et al. 2009).

Gemäss Umfragen, die vorgängig zur epidemiologischen Untersuchung durchgeführt wurden, waren für die Felchen aus Bieler und Brienzer See keine bis sehr geringe Prävalenzen veränderter Gonaden zu erwarten. Die praktische Untersuchung zeigte aber überraschenderweise, dass in beiden Seen zwar geringere Prävalenzen als im Thuner See vorlagen, aber immerhin noch bis zu 30\% der Felchen morphologisch veränderte Gonaden aufwiesen (Abb. 2). Nun
Abb. 2 Gonadenveränderungen bei Felchen aus dem Thuner See, dem Brienzer See und dem Bieler See. Gezeigt sind die Prävalenzen aller Veränderungen (Verwachsungen, Asymmetrien, usw.) von männlichen Felchen aus den drei Seen. Die Felchen wurden im Rahmen eines mehrjährigen monatlichen Monitorings gesammelt, bei dem jeden Monat 25 Fische aus jedem der drei Seen untersucht werden. Daten aus Bittner et al. (2009)
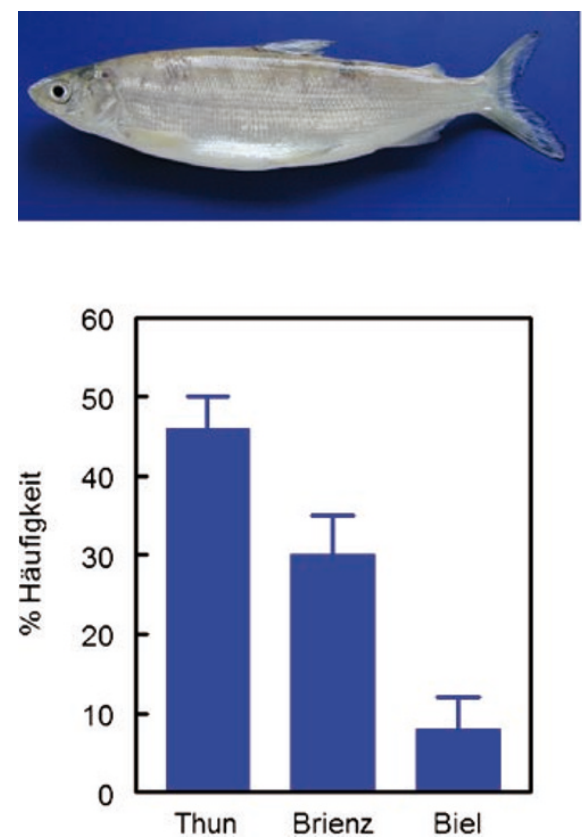
könnte man diesen Befund so interpretieren, dass der gleiche induzierende Faktor in allen drei Seen vorliegt, denn der Prävalenzbefund als solcher erlaubt noch keine Aussage, ob der hohe Anteil veränderter Gonaden ein natürliches oder ein induziertes Phänomen ist. Die genauere Analyse der Daten ergab jedoch, dass bestimmte morphologische Veränderungen, z. B. Asymmetrien, in vergleichbarer Häufigkeit in allen drei Seen auftraten, wohingegen andere Variationen wie z. B. die Verwachsung der Gonade mit der lateralen Muskulatur, im Bieler und Brienzer See sehr selten waren, und überwiegend bei Felchen aus dem Thuner-See auftraten (Bittner et al. 2009). Der Unterschied zwischen Felchen aus dem Thuner See und jenen aus Bieler und Brienzer See liegt somit nicht darin, dass die Thuner Felchen veränderte Gonaden aufweisen und die Felchen aus den anderen Seen bestenfalls eine geringe Hintergrundvariabilität besitzen, sondern darin, dass die Thuner-See-Felchen eine stärkere und anders geartete Variabilität zeigen als die Felchen aus Bieler und Brienzer See. Diese Aussage wurde aber erst durch die umfassende Studie zur Grundvariabilität der Gonadenmorphologie bei Felchen möglich, deren Ergebnisse nahe legten, dass Felchen aus den voralpinen Seen natürlicherweise einen hohen Anteil an bestimmten morphologischen Veränderungen der Gonaden aufweisen. Derartiges Wissen ist essenziell für die Unterscheidung ,natürlich versus induziert“, denn damit lässt sich einerseits eine Fehlinterpretation der hohen Prävalenzen im Bieler und Brienzer See als induzierte Veränderungen vermeiden, und andererseits lässt sich die im Thuner See vorhandene zusätzliche, offensichtlich induzierte Variabilität klar von der Grundvariabilität abtrennen.

Das Beispiel der Thuner-See-Felchen illustriert, wie gering unser Wissen zur Variabilität morphologischer Merkmale bei freilebenden Tierpopulationen ist. Darüber hinaus macht es deutlich, wie sehr die Interpretation von Beobachtungen an Wildtierpopulationen von unserem Wissensstand zu diesen Arten abhängt. Ohne hinreichende Kenntnis der Grundvariabilität ist die Nutzung von biologischen Merkmalen als Biomarker letztlich nicht zuverlässig möglich. Dabei können wir davon ausgehen, dass die meisten biologischen Merkmale - selbst die oft als invariabel empfundenen morphologischen Merkmale - ein hohes Mass an Variabilität aufweisen, denn dies ist essenziell für das Überleben der Art in einer variablen Umwelt. Ein Problem in der Generierung von mehr Informationen zur Variabilität biologischer Merkmale ist dabei, dass in der heutigen Forschungslandschaft nur wenige Ressourcen für die Durchführung derartig „,deskriptiver" Forschung zur Verfügung stehen.

\section{Schlussbemerkungen}

In der Ökotoxikologie tendieren wir dazu, (innerartliche) Variabilität als unerwünschte Störgrösse zu sehen, und versu- chen, sie soweit als möglich zu minimieren oder zu eliminieren. Dieser Ansatz führt bei Labortests zur Tendenz, genetisch verarmte Populationen einzusetzen, was die Übertragbarkeit auf das Freiland einschränkt (Soares et al. 1992). Ähnlich verhalten wir uns bei der Modellierung von Populationen häufig so, als beständen Populationen aus Klonen anstatt aus unterschiedlichen Individuen (Koijman 1998), was die Aussagekraft der Modellierungsergebnisse einschränkt. Schliesslich arbeiten wir im Freiland oft mit ungenügenden Stichprobengrössen, die die Variabilität unzureichend abbilden bzw. die uns wegen der bei der Planung der Untersuchungsstrategie nicht hinreichend in Betracht gezogenen Variabilität keine Aussage zur Stressoreinwirkung erlauben (Underwood 1994; Sumpter und Johnson 2005). Dabei vernachlässigen wir, dass genotypische und phänotypische Variabilität die Grundlage der Anpassungsfähigkeit von Arten an Umweltstress bilden (Day und McPhail 1996; Fordyce 2006). Die Ökotoxikologie könnte davon profitieren, wenn sie Variabilität von biologischen und ökologischen Merkmalen nicht nur als Störgrösse behandelt, sondern die darin enthaltene Information für das Verständnis von umweltstressinduzierten Effekten nutzt (van Straalen 2003; Charmantier et al. 2008).

\section{Literatur}

Au DWT (2004) The application of histo-cytopathological biomarkers in marine pollution monitoring: a review. Mar Poll Bull 48: $817-834$

Behrens A, Segner H (2005) Cytochrome P4501A induction in brown trout exposed to small streams of an urbanised area: results of a five-year-study. Environ Poll 136:231-242

Bernet D, Schmidt H, Meier W, Burkhardt-Holm P, Wahli T (1999) Histopathology in fish: proposal for a protocol to assess aquatic pollution. J Fish Dis 22:25-34

Bernet D, Wahli T, Kueng C, Segner H (2004) Frequent and unexplained gonadal abnormalities in whitefish (central alpine Coregonus sp.) from an alpine oligotrophic lake in Switzerland. Dis Aquat Org 61:137-148

Bernet D, Liedtke A, Bittner D, Eggen RIL, Kipfer S, Küng C, Largiader CR, Suter MJ-F, Wahli T, Segner H (2008) Gonadal Malformations in Whitefish from Lake Thun: Defining the Case and Evaluating the Role of EDCs. Chimia 62:383-388

Bittner D, Bernet D, Wahli T, Segner H, Küng C, Largiader CR (2009) How normal is abnormal? - Discriminating between deformations and natural variation in gonad morphology of European whitefish Coregonus lavaretus from Swiss lakes. J Fish Biol, accepted

Böhm R, Hanke W, Segner H (1994) The sequential restoration of plasma metabolite levels, liver composition and liver structure in refed carp, Cyprinus carpio. J Comp Physiol B 164:32-41

Brakefield PM (1997) Phenotypic plasticity and fluctuating asymmetry as responses to environmental stress in the butterfly Bicyclus anymnana. In: Bijlsma R, Loeschke V (eds) Environmental stress, adaptation and evolution. Birkhäuser, Basel, pp 65-78

Braunbeck T (1998) Cytological alterations in fish hepatocytes following in vivo and in vitro sublethal expsoure to xenobiotics structural biomarkers of environmental contamination. In: Braunbeck T, Hinton DE, Streit B (eds) Fish Ecotoxicology. Birkhäuser, Basel, pp 61-140

Braunbeck T, Gorgas K, Storch V, Völkl A (1987) Ultrastructure of hepatocytes of golden ide (Leuciscus idus melanotus L., Cy- 
pinidae: Teleostei) during thermal adaptation. Anat Embryol 175:303-313

Braunbeck T, Storch V, Nagel R (1989) Sex-specific reaction of liver ultrastructure in zebrafish (Brachydanio rerio) and trout (Salmo gairdneri) after prolonged exposure to 4-chlorophenol. Aquat Toxicol 14:185-202

Braunbeck T, Görge G, Storch V, Nagel R (1990) Hepatic steatosis in zebrafish (Brachydanio rerio) induced by long-term exposure to gamma-hexachlorcyclohexane. Ecotox Environ Safety 19: 355-374

Brown EAR, Scott DBC (1988) A second hermaphrodite specimen of Coregonus lavaretus from Loch Lomond, Scotland. J Fish Biol 33:957-958

Burkhardt-Holm P, Oulmi Y, Schroeder A, Storch V, Braunbeck T (1998) Toxicity of 4-chloroaniline in early life stages of zebrafish (Danio rerio). II Cytopathology and regerenration of liver and gills after prolonged exposure to water-borne 4-chloroaniline. Arch Environ Contam Toxicol 37:85-102

Charmantier A, McCleery RH, Cole LR, Perrins C, Kruuk LEB, Sheldon BC (2008) Adaptive phenotypic plasticity in response to climate change in a wild bird population. Science 320:800-803

Clarke GM (1993) Fluctuating asymmetry of invertebrate populations as a biological indicator of environmental quality. Environ Poll 82:207-211

Couch JA (1975) Histopathological effects of pesticides and related chemicals on the liver of fishes. In: Ribelin WE, Migaki G (eds) The Pathology of Fishes. University of Wisconsin Press, Madison, pp 559-584

Day T, McPhail JD (1996) The effect of behavioural and morphological plasticity on foraging efficiency in the threespine stickleback (Gasterosteus sp.). Oecologia 108:380-388

Desbrow C, Routledge EJ, Brighty GC, Sumpter JP, Waldock M (1998) Identification of estrogenic chemicals in STW effluents. I. Chemical fractionation and in vitro biological screening. Environ Sci Technol 32:1549-1558

Dittbrenner N, Lazzara R, Köhler H-R, Mazzia C, Capowiez Y, Triebskorn R (2008) Heat tolerance in mediterranean land snails: Histopathology after exposure to different temperature regimes. J Moll Stud, in press

Dittrich D, Krieger H, Rumpf S, Segner H, Wester P, Fournie JW, Wolf JJ, van der Ven L, Gimeno S (2009) Histological analysis of endocrine disrupting effects in small laboratory fish. Wiley, New York, in press

Downes BJ, Barmuta LA, Fairweather PG, Faith DP, Keough MJ, Lake PS, Mapstone BD, Quinn GP (2002) Monitoring Ecological Impacts. Cambridge University Press, Cambridge

Eakin RM (1972) Structure of invertebrate photoreceptors. In: Handbook of Sensory Physiology, vol 7. Springer, Berlin, pp 625-684

Fordyce JA (2006) The evolutionary consequences of ecological interactions mediated through phenotypic plasticity. J Exp Biol 209:2377-2383

Gerken J, Sordyl H (2002) Intersex in feral marine and freshwater fish from northeastern Germany. Mar Environ Res 54:651-655

Gernhöfer M, Pawert M, Schramm M, Müller E, Triebskorn R (2001) Ultrastructural biomarkers as tools to characterize the health status of fish in contaminated streams. J Aquat Ecosyst Stress recov $8: 241-260$

Gimeno S, Komen H, Gerritsen AGM, Bowmer T (1998) Feminization of young males of the common carp, Cyprinus carpio, exposed to 4-tert-pentylphenol during sexual differentiation. Aquat Toxicol 43:77-92

Gray MA, Metcalfe CD (1997) Induction of testis-ova in Japanese medaka (Oryzias latipes) exposed to p-nonylphenol. Environ Toxicol Chem 16:1082-1086

Grim KC, Wolfe M, Hawkins W, Johnson R, Wolf J (2007) Intersex in Japanese medaka (Oryzias latipes) used as negative controls in toxicological bioassays: a review of 54 cases from 41 studies. Environ Toxicol Chem 26:1636-1643

Hinton DE, Klaunig JE, Lipsky MM (1978) PCB-induced alterations in teleost liver: a model for environmental disease in fish. Mar Fish Rev 40:47-50

Hinton DE, Lantz RC, Hampton JA, McCuskey PR, McCuskey RS (1987) Normal versus abnormal structure: considerations in morphological responses of teleosts to pollutants. Environ Health Perspect 71:139-146

Hinton DE, Segner H, Au DWT, Kullman SW, Hardman RC (2008). Liver toxicity. In: DiGiulio RT, Hinton DE (eds) The Toxicology of Fishes. Taylor and Francis Publishers, Boca Raton, FL, pp $327-400$

Jamieson BGM (1986) Onychophoran-euclitellate relationships: evidence from spermatozoal ultrastructure. Zool Scripta 15: $141-155$

Jobling S, Nolan M, Tyler CR, Brighty G, Sumpter JP (1998) Widespread sexual disruption in wild fish. Environ Sci Technol 32:2498-2506

Johnston IA (2006) Environment and plasticity of myogenesis in teleost fish. J Exp Biol 209:2249-2264

Kinnison MT, Unwin MJ, Jara F (2000) Macroscopic intersexuality in salmonid fishes. New Zealand J Mar Freshwat Res 34:125-134

Köhler A (2004) The gender-specific risk to liver toxicity and cancer of flounder (Platichthys flesus) at the German Wadden Sea coast. Aquat Toxicol 70:257-276

Köhler A, Deisemann H, Lauritzen B (1992) Histological and cytochemical indices of toxic injury in the liver of dab, Limanda limanda. Mar Ecol Progr Ser 91:141-153

Körner O, Vermeirssen ELM, Burkhardt-Holm P (2007) Reproductive health of brown trout inhabiting Swiss rivers with declining fish catch. Aquat Sci 69:26-40

Kipfer S, Segner H, Wenger M, Wahli T, Bernet D (2009) Long-term estrogen exposure of whitefish (Coregonus lavaretus) induces intersex but not the Lake Thun-typcial gonad malformations. Dis Aquat Org, accepted

Kooijman SALM (1998) Process-oriented description of toxic effects. In: Schüürmann G, Markert B (eds) Ecotoxicology. Wiley, New York pp 484-520

Lange U, Saborowski R, Siebers D, Buchholz F, L Karbe (1998) Temperature as a key factor determining the regional variability of the xenobiotic-inducible ethoxyresorufin-O-deethylase activity in the liver of dab (Limanda limanda). Can J Fish Aquat Sci 55:328-338

McIntyre PB, Baldwin S, Flecker AS (2004) Effects of behavioural and morphological plasticity on risk of predation in a neotropical tadpole. Oecologia 141:130-138

McLachlan JA (2001) Environmental signaling: what embryos and evolution teach us about endocrine disrupting chemicals. Endocr Rev 22:319-341

Myers MS, Stehr CM, Olson OP, Johnson LL, McCain BB, Chan SL (1994) Relationships between toxicopathic hepatic lesions and exposure to chemical contaminants in English sole (Parophrys vetulus), starry flounder (Platichthys stellatus) and white croaker (Genyonemus lineatus) from selected marine sites on the Pacific coast, USA. Environ Health Perspect 102:200-215

Oehlmann J, Fiorini P, Stroben E, Markert B (1996) Tributyltin effects on Ocinebrina aciculata (Gastropoda: Muricidae): imposex development, sterilization, sex change and population decline. Sci Total Environ 188:205-223

Schwaiger J, Wanke R, Adam S, Pawert M, Honnen W, Triebskorn R (1997) The use of histopathological indicators to evaluate contaminant-induced stress in fish. J Aquat Ecosyst Stress Recov 6:75-86

Segner H (1987) Response of fed and starved roach, Rutilus rutilus, to sublethal copper contamination. J Fish Biol 30:423-437 
Segner H (2009) Zebrafish (Danio rerio) as a model organism for investigating endocrine disruption. Comp Biochem Physiol, Part C, 149:187-195

Segner H, Möller H (1984) Electron microscopical investigations on starvation-induced liver pathology in flounders, Platichthys flesus. Mar Ecol Progr Ser 19:193-196

Segner H, Braunbeck T (1990) Adaptive changes of liver composition and structure in golden ide during winter acclimatization. J Exp Zool 255:171-185

Soares AMVM, Baird DJ, Calow P (1992) Interclonal variation in the performance of Daphnia magna Strauss in chronic bioassays. Environ Toxicol Chem 11:1477-1483

Söderman F, van Dongen S, Pakkasmaa S, Merilä J (2007) Environmental stress increases skeletal fluctuating asymmetry in the moor frog Rana arvalis. Oecologia 151:593-604

Storch V (1979) Contributions of comparative ultrastructural research to problems of invertebrate evolution. Am Zool 19:637-645

Storch V (1982) Der Einfluss der Ernährung auf die Ultrastruktur der großen Zellen in den Mitteldarmdrüsen terrestrischer Isopoda (Armadillium vulgare, Porcellio scaber). Zoomorphology 100:131-142

Storch V (1985) Die Zelle als Spiegel der Umwelt. Umschau 1:21-23

Storch V, Stählin W, Juario JV (1983) Effects of different diets on the ultrastructure of hepatocytes of Chanos chanos fry (Chanidae:Teleostei): an electron microscopic and morphometric analysis. Mar Biol 74:101-104

Storch V, Welsch U (1989) Ultrastruktur und Evolution. Naturwiss Rundschau 42:100-106

Sumpter JP, Johnson AC (2005) Lessons from endocrine disruption and their application to other issues concerning trace organics in the aquatic environment. Environ Sci Technol 39:4321-4332
Triebskorn R Künast C (1990) Ultrastructural changes in the digestive system of Deroceras reticulatum (Mollusca: Gastropoda) induced by lethal and sublethal concentrations of the carbamate molluscicide Cloethocarb. Malacologia 32:89-106

Underwood AJ (1994) On beyond BACI: sampling designs that might reliably detect environmental disturbances. Ecol Applic 4:31-35

van Aerle R, Nolan M, Jobling S, Christiansen LB, Sumpter JP, Tyler CR (2001) Sexual disruption in a second species of wild cyprinid fish, the gudgeon (Gobio gobio) in United Kingdom freshwaters. Environ Toxicol Chem 20:2841-2847

van Straalen NM (2003) Ecotoxicology becomes stress ecology. Environ Sci Technol 37:324A-330A

Vine E, Shears J, van Aerle R, Tyler CR, Sumpter JP (2005). Endocrine (sexual) disruption is not a prominent feature in the pike (Esox lucius), a top predator, living in English waters. Environ Toxicol Chem 24:1436-1443

Vogt G, Quinitio ET, Pascual FP (1986). Leucaena leucoephala leaves in formulated feed for Penaeus monodon: a concret example of the application of histology in nutrition research. Aquaculture 59:209-234

Vogt G (1987) Monitoring of environmental pollutants such as pesticides in prawn aquaculture by histological diagnosis. Aquaculture 67:157-164

Walker CH (1998) Biomarker strategies to evaluate the environmental effects of chemicals. Environ Health Perspect 106 (suppl 2): 613-620

Watanabe KH, Jensen KM, Orlando EF, Ankley GT (2007) What is normal? A characterization of the values and variability in reproductive endpoints of the fathead minnow, Pimephales promelas. Comp Biochem Physiol 146C:348-356 